\title{
Supplementary material-3
}

\section{Coordination geometry around the metal ion optimization of the model:}

After the development of best packing quality final model for $P$. aeruginosa LpxC, co-ordinate of $\mathrm{Zn}^{+2}$ and water molecule are added to final models using MOE and further, their structures were optimized using gradient $0.01 \mathrm{Kcal} / \mathrm{mol} \AA$ using MMFF94 force field and PM3 Hamiltonian in MOE. The optimized geometric parameters abounds the metal ions were used for optimization of three dimensional structure of both proteins. Models were further refined by energy minimization to remove any steric clashes of the side chains with each other and/or with backbone atoms. The model was first subjected to a highly tethered series of conjugate gradient minimization steps. The coarse, medium and fine minimization options all use the Truncated Newton optimization algorithm, with RMS gradient tests of 10,1 and $0.1 \AA$ respectively. The Medium option was selected for the modeling.

Validation:

In the homology model, coordinates of $\mathrm{Zn}^{+2}$ atoms and water in active site were mimicked from the crystal structure of template (Figure 1). The distances measured between the atom-pairs are given in table 1 . This validate our protocol for addition of metal ion and water molecule to proteins. 
Figure 1

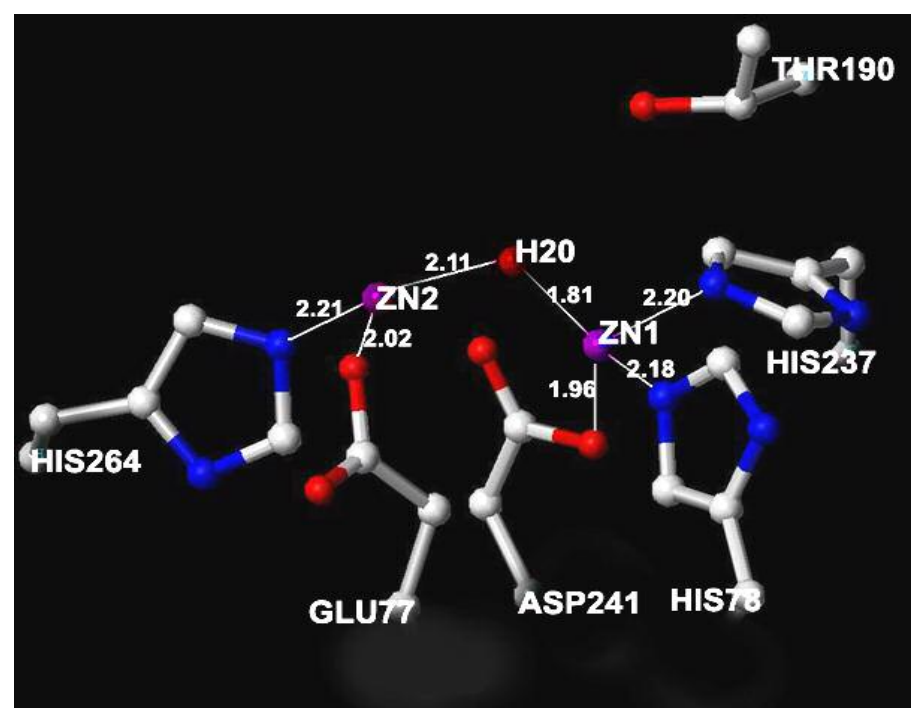

Table-2

\begin{tabular}{lccc}
\hline & & \multicolumn{2}{c}{ Distance $(\mathbf{A})$} \\
Atom1 & Atom2 & $\begin{array}{c}\text { Crystal structure- } \\
\text { PaLpxC }\end{array}$ & Homology \\
& & Template) & Model \\
Zn1 & NE2(His78) & 2.08 & 2.18 \\
Zn1 & NE2(His237) & 2.17 & 2.2 \\
Zn1 & OD1(Asp241) & 1.99 & 1.959 \\
Zn1 & O(H2O) & 1.80 & 1.804 \\
Zn2 & O(H2O) & 2.11 & 2.105 \\
Zn2 & OE2(Glu77) & 1.98 & 2.02 \\
Zn2 & NE2(His264) & 2.07 & 2.21 \\
\hline
\end{tabular}

